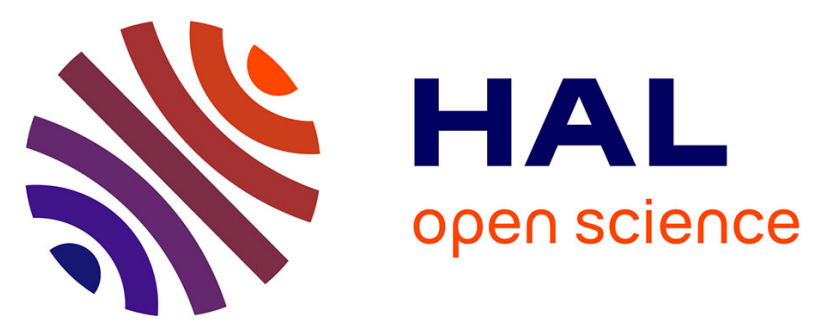

\title{
L'analyse des politiques agricoles: l'apport de l'équilibre général calculable
}

\author{
Alexandre Gohin, Herve Guyomard, Chantal Le Mouël
}

\section{To cite this version:}

Alexandre Gohin, Herve Guyomard, Chantal Le Mouël. L'analyse des politiques agricoles: l'apport de l'équilibre général calculable. Journée: L'agriculture dans l'économie. Modélisations macroéconomiques, modèles d'équilibre général calculable, Apr 1998, Paris, France. 17 p. hal-02837721

\section{HAL Id: hal-02837721 \\ https://hal.inrae.fr/hal-02837721}

Submitted on 7 Jun 2020

HAL is a multi-disciplinary open access archive for the deposit and dissemination of scientific research documents, whether they are published or not. The documents may come from teaching and research institutions in France or abroad, or from public or private research centers.
L'archive ouverte pluridisciplinaire HAL, est destinée au dépôt et à la diffusion de documents scientifiques de niveau recherche, publiés ou non, émanant des établissements d'enseignement et de recherche français ou étrangers, des laboratoires publics ou privés.

\section{(ㅇ)(1) $\$$}

Distributed under a Creative Commons Attribution - NonCommercial - NoDerivatives $\mid 4.0$ 
INSTITUT NATIONAL DE LA RECHEGLLE AGHUivuiviluU.

\title{
L'AGRICULTURE DANS L'ECONOMIE
}

modélisations macro-économiques, modèles d'équilibre général calculable

JOURNEE SFER-INRA

PARIS, 29 AVRIL 1998

\section{L'ANALYSE DES POLITIQUES AGRICOLES : L'APPORT DE I'EQUILIBRE GENERAL CALCULABLE}

\author{
Alexandre Gohin, Hervé Guyomard, Chantal Le Mouël \\ INRA-ESR, équipe PAM, Rennes
}

\section{Introduction}

Depuis le premier modèle d'équilibre général calculable (MEGC) développé par Johansen (1960), modèle centré sur l'économie norvégienne, et surtout depuis la découverte par Scarf (1967) d'un algorithme de résolution numérique de problèmes spécifiés dans un cadre d'équilibre général, la construction et l'utilisation des MEGCs ont connu un essor considérable. De manière générale, les thèmes étudiés dans un cadre d'équilibre général calculable peuvent être classés dans trois groupes : des problèmes sectoriels, essentiellement pour l'agriculture et l'énergie, des problèmes relatifs aux politiques commerciales et aux échanges, et des problèmes liés aux réformes de la fiscalité et des finances publiques. Plus récemment, plusieurs MEGCs ont été développés pour étudier des questions environnementales (Beaumais et Schubert, 1994).

Un MEGC est, comme tout modèle, bien adapté pour apporter des éléments de réponse aux questions spécifiques pour lesquelles il a été initialement construit. La structure du modèle, en termes de découpage géographique, de désagrégation des secteurs d'activité et des ménages, de choix des formes fonctionnelles à l'offre et à la demande, de représentation explicite des instruments de politique économique, etc. dépend donc, très fortement, de la nature des questions posées. De manière générale, 
la complexité "technique" d'un MEGC a tendance à décroître quand sa dimension, mesurée en termes de zones géographiques distinguées et de secteurs d'activité différenciés, augmente. De multiples facteurs expliquent ce trade-off, notamment les difficultés de résolution numériques de grands modèles non linéaires, de définition d'une base initiale de données cohérente sous la forme d'une matrice de comptabilité sociale (MCS) et d'interprétation des résultats (François et Shiells, 1994). Dans le cas des MEGCs "sectoriels", il y a notamment un trade-off clair entre l'augmentation du nombre de secteurs d'activité et la modélisation détaillées des caractéristiques technologiques et institutionnelles de ces derniers.

L'objectif de cet article est double. Dans une première section, nous définissons ce qu'est un modèle d'équilibre général calculable en montrant que ce dernier peut être considéré, d'une part comme un prolongement des modèles à coefficients fixes par la généralisation du tableau input-output à l'ensemble de l'économie considérée et par la prise en compte des ajustements par les prix, d'autre part comme un prolongement des modèles d'équilibre partiel multi-marchés par la prise en compte de tous les marchés (des biens et des facteurs primaires de production), le respect de toutes les identités comptables macro-économiques et la modélisation de tous les flux correspondants. Dans une seconde section, nous montrons l'intérêt de la modélisation en équilibre général calculable de problèmes agricoles. De façon plus précise, nous illustrons, sur la base d'exemples concrets, l'importance des allocations factorielles entre secteurs et l'importance des effets indirects liés à la vérification des identités macro-économiques. Nous discutons également les problèmes de la cohérence théorique des modèles appliqués et de la quantification des paramètres de comportement.

\section{Modélisation en équilibre partiel multi-produits versus équilibre général calculable}

i) Du tableau input-output à la matrice de comptabilité sociale et à la modélisation en équilibre d'équilibre général calculable

Dans la forme, un modèle d'équilibre général calculable est un prolongement naturel des modèles input-output à coefficients fixes qui capturent les liens intersectoriels ayant pour origine les flux de consommations intermédiaires entre secteurs productifs. Les multiplicateurs input-output calculés à partir d'une matrice input-output permettent d'évaluer les impacts d'une variation de la demande finale 
en un bien particulier sur tous les secteurs d'activité de l'économie considérée en supposant que la production de chaque bien est commandée par la demande et qu'il existe une surcapacité de production dans chaque secteur d'activité de sorte qu'un accroissement exogène de la demande finale en un bien peut toujours être satisfait en augmentant la production des biens à des prix inchangés. ' L'extension du tableau input-output à l'ensemble de l'économie permet de construire une matrice de comptabilité sociale qui retrace tous les flux de cette économie pour une année de référence sous la forme d'un tableau carré, équilibré et divisé en un certain nombre de comptes identique en lignes et en colonnes. Les comptes généralement distingués sont ceux des activités, des biens, des facteurs primaires de production, des institutions, du capital et du reste du monde. Pour un compte donné, la ligne représente les ressources qui équilibrent les dépenses réparties en colonne. L'extension de la modélisation inputoutput à la MCS permet de calculer les multiplicateurs généraux à prix fixes qui mesurent les impacts à prix fixes des variations des éléments des comptes de "demande" supposés exogènes (le plus souvent, l'institution gouvernementale, le capital et/ou le reste du monde) sur les éléments des autres comptes supposés endogènes. ${ }^{2}$ Comme leurs homologues input-output, les multiplicateurs généraux de demande à prix fixes ne capturent que les effets de demande. Ils ne sont donc adaptés qu'à des situations keynésiennes caractérisées par des prix fixes, des capacités productives en excès dans tous les secteurs d'activité et l'absence de substitutions à l'offre et à la demande. ${ }^{3}$ Ce cadre d'analyse n'est donc pas adapté pour représenter le fonctionnement réel d'une économie où les ajustements par les prix jouent un rôle majeur et où les possibilités de substitution à l'offre et à la demande sont importantes. La construction d'un modèle non linéaire où les prix et les quantités sont déterminés simultanément, de manière endogène, apparaît alors clairement. Tel est précisément l'objet de la modélisation en équilibre général $\mathrm{c}^{-1}$ culable.

\footnotetext{
${ }^{1}$ Midmore (1987), par exemple, utilise la modélisation input-output pour analyser la place de la branche agricole dans l'économie du Pays de Galles.

${ }^{2}$ Roberts (1994), par exemple, utilise les multiplicateurs généraux de demande à prix fixes pour analyser la place du complexe agricole et agro-alimentaire dans l'économie du Royaume-Uni.

${ }^{3}$ Le calcul des multiplicateurs généraux de demande à prix fixes suppose que les différentes dépenses d'un compte donné sont une proportion fixe de la dépense totale reportée en fin de ligne dans la MCS. Il est possible d'analyser des chocs d'offre de manière similaire en construisant des multiplicateurs généraux d'offre à prix fixes qui reposent sur l'hypothèse miroir selon laquelle les différentes ressources d'un compte donné sont une proportion fixe de la ressource totale reportée en bas de colonne dans la MCS (Roberts, 1994).
} 
ii) Les modèles d'équilibre général calculable: une généralisation à l'ensemble de l'économie des modèles d'équilibre partiel multi-marchés

Même si les modèles d'équilibre général calculable recouvrent des modélisations en pratique très différentes, notamment en termes d'hypothèses relatives au fonctionnement des marchés des facteurs primaires de production et/ou de règles de bouclage macro-économiques, deux caractéristiques communes permettent de les définir simplement (Schubert, 1993). En premier lieu, ce sont des modèles d'équilibre général qui, le plus souvent, dérivent explicitement les comportements des agents en matière d'offre et de demande de programmes d'optimisation, calculent les prix relatifs sur tous les marchés qui assurent les équilibres de ces derniers, et déterminent les allocations sectorielles des facteurs primaires de production entre secteurs d'activité et la répartition qui en découle. ${ }^{4}$ En second lieu, ce sont des modèles calculables qui permettent une analyse quantitative de problèmes économiques qui sont parfois difficiles, voire impossibles, à étudier sur la seule base d'une modélisation théorique.

Dans l'esprit, un MEGC est donc proche d'un modèle d'équilibre partiel multi-marchés dans lequel les décisions des agents réagissent (essentiellement) aux variations des prix relatifs et les marchés assurent les équilibres entre les offres et les demandes de biens. Par rapport à un modèle d'équilibre partiel multi-marchés, un MEGC incorpore cependant des éléments additionnels macro-économiques qui assurent sa cohérence théorique globale et garantissent que tous les flux de l'économie considérée sont comptabilisés et équilibrés. En particulier, il assure l'équilibre du budget du gouvernement et des administrations publiques, l'équilibre de la balance des paiements, et l'égalité entre l'épargne et l'investissement. La cohérence comptable de la modélisation en équilibre général calculable (au sens

\footnotetext{
${ }^{4}$ De manic̀re générale, les premiers MEGCs développés avaient pour objectif d'appliquer la structure théorique, abstraite, de l'équilibre général walrassien (Arrow et Debreu, 1954 ; Arrow et Pratt, $1971 ; \ldots$ ). à des économies réelles où prix et quantités s'ajustent simultanément, dans un cadre de concurrence pure et parfaite, de façon à équilibrer tous les marchés des biens et des facteurs primaires de production. Les modèles plus récents s'éloignent du cadre souvent trop restrictif de l'équilibre walrassien en incorporant différents éléments additionnels comme des structures imparfaites de marché, des possibilités de rationnements à l'offre ou à la demande, des rigidités des prix des biens et des facteurs primaires de production, etc. Le problème qui se pose alors est celui du trade-off entre le réalisme du modèle et le respect de sa cohérence théorique dans la mesure où ces éléments complémentaires qui, sans nul doute, sont nécessaires pour décrire avec réalisme le fonctionnement de l'économie considérée sont parfois introduits de manière ad-hoc, détruisant alors la cohérence théorique sous-jacente de l'ensemble.
} 
où, par exemple, les ménages ne peuvent pas consommer plus qu'ils ne gagnent et où la même unité de travail ne peut pas être simultanément employée dans deux secteurs d'activité distincts) est, indiscutablement, un des intérêts essentiels de l'équilibre général par rapport à une approche en équilibre partiel. La cohérence théorique globale de la modélisation en équilibre général (au sens où toutes les fonctions de comportement sont explicitement dérivées de programmes d'optimisation et où tous les flux de l'économie sont pris en compte sous la forme d'identités comptables) est un second point fort de l'approche. Il faut cependant noter que les modèles d'équilibre partiel multi-marchés sont, dans de nombreux cas, construits sur un principe théorique micro-économique identique, i.e., la dérivation des fonctions de réaction des offres et des demandes de biens des programmes d'optimisation des agents pris en compte. ${ }^{5}$ Les modèles d'équilibre général calculable présentent un avantage additionnel dans la mesure où la vérification de la loi de Walras garantit la cohérence théorique globale de la construction. En pratique, cette vérification prend la forme de l'indépendance des résultats d'une simulation donnée vis-à-vis de l'équation omise, i.e., du marché non explicitement modélisé.

iii) De l'équilibre partiel à l'équilibre général : l'adjonction d'un secteur résiduel, la modélisation des allocations factorielles et des identités comptables macro-économiques

De manière générale, le passage d'un cadre d'analyse en équilibre partiel mono-produit ou multiproduits à un cadre d'analyse en équilibre général requiert le bouclage du modèle par la prise en compte de toutes les liaisons, i.e., des flux, qu'entretiennent le ou les marchés considérés avec tous les autres produits et les facteurs primaires de production. La première étape, i.e., la prise en compte des autres biens de l'économie considérée, consiste à agrandir la fr _atière originale de transformation des produits agricoles en incluant un agrégat qui regroupe tous les autres biens qui ne sont pas explicitement différenciés. Cette approche est utilisée, par exemple, par Horridge et Pearce (1988) qui ajoutent un secteur d'activité mono-produit résiduel au modèle de Tyers et Anderson (1986), modèle

\footnotetext{
5 Cependant, de nombreux modèles d'équilibre partiels multi-produits correspondent à des formes réduites et/ou à des spécifications ad-hoc, ce qui rend difficile l'interprétation des résultats et engendre des risques d'incohérences théoriques dans la mesure où il est dur, voire impossible, de "remonter" aux hypothèses en termes de préférences des consommateurs, de technologies de production ou de mobilité des facteurs primaires de production.
} 
d'équilibre partiel centré sur les céréales, les produits de l'élevage et le sucre. Horridge et Pearce modifient également les fonctions de long terme d'offre et de demande des différents biens de façon à ce que ces dernières puissent explicitement être dérivées de comportements d'optimisation des producteurs et des consommateurs. En d'autres termes, Horridge et Pearce s'assurent de la cohérence théorique globale de leur modèle en corrigeant et en complétant les matrices initiales des élasticités de long terme d'offre et de demande par le respect des contraintes de symétrie, d'homogénéité et de concavité/convexité. Les conséquences d'une libéralisation des échanges agricoles sont similaires dans le modèle d'équilibre partiel de Tyers et Anderson et dans celui de Horridge et Pearce dans la mesure où les effets dus à l'équilibre général se réduisent à des effets des termes de l'échange. Ces derniers sont très faibles parce que les fonctions de demande d'exportation du bien résiduel, supposé homogène, sont très inélastiques. Il ne faut pourtant pas conclure que les modèles d'équilibre partiel fournissent toujours une bonne "approximation" du résultat qui serait obtenu, à long terme dans le cas particulier de Horridge et Pearce, dans un cadre d'équilibre général. Il ne faut pas conclure ainsi car le caractère général du modèle de Horridge et Pearce n'est en réalité que très partiel dans la mesure où les mouvements des facteurs primaires de production entre les différents secteurs agricoles différenciés, et entre ces derniers et le reste de l'économie, ne sont pas pris en compte. En d'autres termes, le passage complet de l'équilibre partiel à l'équilibre général implique non seulement l'élargissement de la frontière initiale de transformation des productions à l'ensemble des biens de l'économie, mais également l'extension de cette frontière de transformation à la définition de la frontière des possibilités de production qui intègre tous les biens et les facteurs primaires de production (Hertel, 1990b). Il faut en outre "fermer" le système étudié, i.e., équilibrer toutes les identités comptables macro-économiques de l'économie considérée et définir les règles de bouclage correspondantes.

En pratique, le problème de l'économiste agricole appliqué est alors le suivant : "à partir de quand" ou, en d'autres termes, pour quelles "classes" de problèmes économiques, le cadre de l'équilibre partiel multi-produits est-il insuffisant et doit-il être complété, voire remplacé, par une modélisation en équilibre général calculable?

\section{Pourquoi utiliser l'approche de l'équilibre général calculable pour étudier des problèmes} agricoles ? 
Il apparaît clairement qu'un intérêt majeur de la modélisation en équilibre général calculable de problèmes agricoles réside dans le fait qu'il est possible de prendre en compte toutes les liaisons entre les secteurs agricoles distingués, les secteurs d'amont et les secteurs d'aval. Dans un cadre d'équilibre partiel, il est en effet souvent difficile de définir une frontière précise qui partitionne les secteurs et les biens en deux ensembles, d'une part les secteurs d'activité et les biens qu'il convient de considérer en raison des liens étroits qu'ils entretiennent avec le secteur et/ou le bien objets de l'étude, d'autre part les secteurs d'activité et les biens qu'il est possible d'ignorer dans la mesure où les effets du scénario considéré sur ces derniers sont supposés, a priori, négligeable. De plus, la modélisation en équilibre général permet de rendre compte, de manière théoriquement cohérente, des mouvements des facteurs primaires de production entre secteurs. Comme le note Hertel (1990a), le soutien accordé aux secteurs agricoles dans les pays développés a pour effet de maintenir des ressources primaires dans l'agriculture à des niveaux vraisemblablement supérieurs à ceux qui seraient observés sans intervention publique. A ce maintien de ressources primaires dans l'agriculture est associé un coût d'opportunité qui n'est que très imparfaitement pris en compte dans un cadre d'équilibre partiel par la spécification de fonctions d'offre des inputs primaires à pente strictement croissante. Enfin, le cadre de l'équilibre général permet de respecter toutes les identités comptables macro-économiques de l'économie considérée ou, en d'autres termes, toutes les égalités entre les ressources et les emplois des différents comptes de la MCS.

i) La nécessaire prise en compte des allocations inter-sectorielles des inputs primaires et des identités comptables macro-économiques

L'importance de la prise en compte des allocations factorielles et des identités comptables macroéconomiques peut être illustrée à partir des travaux de Kilkenny et Robinson (1990). ${ }^{67}$ Ces deux

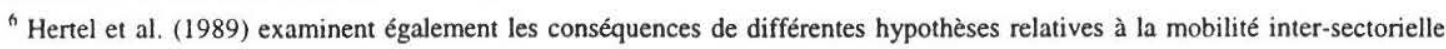
des facteurs primaires de production dans un scénario de suppression unilatérale de la politique américaine de soutien agricole. Leurs conclusions sont qualitativement identiques à celles de Kilkenny et Robinson.

${ }^{7}$ Dans le cas de la France, Gohin et al. (1998) montrent que les effets sur le complexe agro-alimentaire national d'une suppression des mesures de soutien interne dans les secteurs français et communautaire des grandes cultures (céréales et oléoprotéagineux) dépendent étroitement des degrés de mobilité inter-sectorielle des facteurs primaires de production. Plus les
} 
auteurs analysent les conséquences pour l'économie américaine d'une libéralisation, unilatérale ou multilatérale, des programmes de soutien agricole en utilisant un modèle d'équilibre général de l'agriculture américaine, modèle composé de dix secteurs d'activité (trois secteurs agricoles - le lait et la viande, les céréales et les oléagineux, et un secteur agricole résiduel - , cinq secteurs industriels et deux secteurs de services) et calibré sur une MCS de l'année 1986.

Le tableau 1 permet d'analyser les conséquences sur les secteurs agricoles américains d'hypothèses alternatives relatives à la mobilité inter-sectorielle des facteurs primaires de production et à la redistribution des économies budgétaires réalisées dans un scénario de libéralisation unilatérale. Ces conséquences sont mesurées en termes de variations des prix de marché des produits agricoles.

\section{(insérer tableau 1)}

Les hypothèses relatives aux possibilités de mobilité des facteurs primaires de production sont reportées en colonne. Dans le premier cas, noté "terre", les facteurs travail et capital sont fixes et totalement employés dans les différents secteurs d'activité distingués, la terre étant supposée parfaitement mobile entre les différents secteurs qui l'utilisent. Dans le deuxième cas, noté "terretravail", les facteurs terre et travail sont supposés parfaitement mobiles entre secteurs alors que le capital est maintenu fixé et totalement utilisé dans les différents secteurs. Dans le troisième cas, noté "total", les trois facteurs primaires de production sont parfaitement mobiles entre secteurs. Les hypothèses relatives aux règles macro-économiques de bouclage, plus précisément ici les règles de redistribution des économies budgétaires permises par la suppression du programme américain de soutien agricole, sont reportées en ligne. Dans le premier cas, noté "ménages", les économies budgétaires sont redistribuées aux ménages, sous la forme de transferts, de sorte qu'il n'y a pas de variations du déficit budgétaire et du déficit de la balance des paiements. Dans le deuxième cas, noté "investissement", les économies budgétaires réalisées sont épargnées ce qui se traduit par un accroissement de l'investissement en vertu de la détermination de ce dernier par l'épargne (bouclage épargne-investissement néoclassique). Dans le troisième cas, noté "déficits", le déficit budgétaire et les

inputs primaires sont mobiles (respectivement immobiles) entre secteurs, plus les effets en termes de prix des outputs et des inputs sont faibles (respectivement forts) et plus les effets en termes de volumes produits sont forts (respectivement faibles). Les impacts macro-économiques de ce scénario (niveau d'épargne et d'investissement, balance des paiements, etc.) dépendent essentiellement de la manière dont les économies budgétaires réalisées sont réallouées. 
besoins en capitaux étrangers sont réduits d'un même montant, équivalent, au total, aux économies budgétaires réalisées. La dernière ligne du tableau 1 reproduit les résultats du même scénario obtenus à l'aide du modèle d'équilibre partiel SWOPSIM (Roningen, 1986).

De manière générale, il apparaît que les impacts d'une suppression unilatérale du programme américain de soutien agricole sur les secteurs agricoles dépendent très fortement des hypothèses relatives à la mobilité inter-sectorielle des facteurs primaires de production et qu'ils sont pratiquement insensibles à la manière dont les économies budgétaires réalisées sont réallouées. A l'inverse, les impacts de ce scénario sur le reste de l'économie américaine dépendent très fortement de la politique de redistribution des économies budgétaires et assez faiblement des possibilités de mobilités intersectorielles des facteurs primaires de production. ${ }^{8}$ Une lecture en ligne du tableau 1 montre que plus les facteurs primaires de production sont mobiles, plus les baisses des prix de marché des biens agricoles sont faibles. Quand les prix de marché (et les rémunérations factorielles) diminuent dans un secteur d'activité donné, les demandes dérivées des facteurs primaires (et également des consommations intermédiaires) dans ce secteur baissent. Si les facteurs primaires de production sont mobiles entre secteurs, la production diminue par un effet de réallocation des facteurs primaires. Le prix du bien qui équilibre l'offre à la demande augmente alors, ce qui tend à annuler une partie, voir plus (cas du lait dans les hypothèses "terre-travail" et "total"), de la baisse initiale du prix suite à la suppression du soutien.

Une suppression unilatérale du programme de soutien agricole américain conduit à une réallocation des facteurs primaires de production travail et capital hors de l'agriculture vers des secteurs d'activité plus "lucratifs". 9 Cette réallocation factorielle conduit à un accroissement du Produit Intérieur Brut (PIB) américain dans les deux hypothèses macro-économiques où les économies budgétaires sont épargnées et investies (hypothèse macro-économique "investissement") et où les économies budgétaires sont utilisées pour réduire les déficits (hypothèse macro-économique "déficits"). Le gain de PIB est peu sensible à l'hypothèse macro-économique "investissement" versus "déficit", ainsi qu'à l'hypothèse relative à la mobilité inter-sectorielle ou non du facteur primaire capital. Si les facteurs

\footnotetext{
${ }^{8}$ Les impacts sur le reste de l'économie ne sont pas reproduits dans le tableau 1.

${ }^{9}$ Naturellement, cette réallocation n'a lieu que dans les cas où elle est, par hypothèse et pour le facteur primaire considéré, autorisée.
} 
primaires travail et capital sont supposés fixes sectoriellement, le scénario considéré conduit à une diminution du PIB quelle que soit la manière dont les économies budgétaires sont redistribuées. De plus, la variation du PIB est toujours négative sous l'hypothèse d'une redistribution des économies budgétaires aux ménages (hypothèse macro-économique "ménages"). Ce dernier résultat illustre le caractère de second rang du problème étudié. Le critère d'allocation d'équilibre d'un facteur primaire donné est l'égalité du prix nominal (le salaire dans le cas du travail) et de la productivité nominale moyenne marginale. En fonction des distorsions qui existent dans l'économie considérée, les facteurs primaires peuvent alors se réallouer dans des secteurs plus profitables en termes nominaux mais qui ont une production réelle plus faible.

ii) La nécessaire cohérence théorique des modèles agricoles appliqués et le problème de l'évaluation des paramètres de comportement

Le tableau 1 permet également de comparer les résultats, en termes d'effets sur les prix de marché des produits agricoles, obtenus dans ce cadre d'équilibre général avec ceux du modèle d'équilibre partiel SWOPSIM. Il apparaît alors que le modèle d'équilibre général calculable de Kilkenny et Robinson prévoit des baisses des volumes produits plus importants que le modèle d'équilibre partiel SWOPSIM, ce qui se traduit in fine par des baisses des prix de marché des produits agricoles plus faibles en équilibre général. ${ }^{10}$ Cette comparaison montre que l'une des principales faiblesses des modèles d'équilibre partiel multi-marchés spécifiés sous la forme d'équations réduites sans qu'il soit possible de définir explicitement les programmes sous-jacents d'optimisation des agents est la difficulté à identifier les variables implicitement maintenues à des niveaux constants. Dans le cas particulier d'une fonction d'offre d'un producteur agricole mono-produit, il est ainsi souvent difficile, voir impossible, de déterminer si la fonction d'offre spécifiée reflète la réaction optimale du produit considéré aux variations des prix de tous les facteurs ou si les offres de certains inputs (primaires notamment) sont implicitement maintenues constantes pour l'horizon temporel de la simulation. Cette observation souligne la nécessité de s'assurer de la cohérence "micro-économique" théorique de tout modèle, qu'il soit défini dans un cadre d'équilibre général ou dans un cadre d'équilibre partiel. Dans le cas particulier

\footnotetext{
${ }^{10}$ Les écarts s'expliquent aussi par des paramètres comportementaux différents dans les deux modèles, sans qu'il soit possible d'évaluer les implications de ces différences.
} 
d'une fonction d'offre d'un producteur agricole mono-produit ou multi-produits, une solution possible est d'utiliser la théorie de la dualité en dérivant le système complet des offres de produits et des demandes dérivées des facteurs variables d'une fonction de profit restreint. Dans ce cas, la fonction d'offre du produit dépendra des prix des netputs supposés variables et des quantités des netputs supposés fixes. La fixité de certains netputs peut être liée au choix du modélisateur de se situer dans le (très) court terme où les possibilités d'ajustement de certains inputs primaires (cas de la terre et du travail familial agricole, par exemple) sont très réduites, voire nulles, ou à l'environnement économique qui peut imposer la constance de certaines quantités d'outputs (quotas de production) ou d'inputs (quotas d'inputs, gel des surfaces).

Naturellement, le problème de l'estimation des paramètres de comportement, i.e., des élasticités prix et quantités des offres des outputs variables, des demandes dérivées des inputs variables, et des prix virtuels des netputs fixes, se pose de manière identique dans les deux cadres de l'équilibre partiel et de l'équilibre général. Dans le cas de la modélisation en équilibre général calculable, le calibrage des paramètres de comportement consiste, en pratique, à résoudre le modèle à l'envers de façon à retenir des valeurs des paramètres comportementaux inconnus qui permettent de reproduire, en faisant fonctionner le modèle à l'endroit, l'équilibre initial de l'économie considérée, i.e., la MCS. Dans la majorité des situations, l'ensemble des paramètres comportementaux qui satisfait au critère défini cidessus n'est pas unique et le modélisateur doit alors fixer, de manière exogène, certains paramètres pour réduire l'espace des possibles à une solution unique. A l'exception des modèles développés par Jorgenson et ses collaborateurs qui estiment économétriquement tous les paramètres inconnus, le chiffrage des MEGCs est donc déterministe. ${ }^{11}$

\footnotetext{
"Une des faiblesses de la modélisation en équilibre général calculable est l'absence (ou, du moins, la grande difficulté) d'une validation empirique au sens où il est difficile d'évaluer jusqu'à quel point le modèle correspond aux données ou retrace l'évolution passée (Borges, 1986). Cette critique est surtout valables pour les MEGCs statiques qui ne décrivent qu'une "période de temps", par ailleurs très imparfaitement spécifiée. Le problème est moins aigu dans le cas des MEGCs dynamiques dans la mesure où il est alors possible de comparer les résultats fournis par le modèle sur plusieurs périodes et les séries réellement observées (Schubert, 1993). Néanmoins, dans les deux cas, il est impossible de formuler un test statistique permettant de "mesurer la distance" entre la solution fournie par le modèle et la réalité. Le caractère déterministe du calibrage des MEGCs pose, en outre, le problème de la sensibilité des résultats de simulation aux valeurs des paramètres que le modélisateur doit fixer de manière exogène, sur la base d'estimations économétriques "externes" et/ou d'avis d'experts. Des études de sensibilité, mises en œuvre en faisant varier les valeurs des paramètres fixés de manière exogène, permettent de remédier, mais seulement très imparfaitement, à cet inconvénient.
} 
La discussion précédente ne signifie naturellement pas que les modèles d'équilibre partiel multiproduits spécifiées sous une forme réduite ou qui ne respectent pas toutes les implications théoriques des programmes d'optimisation dont ils pourraient être déduits ne présentent aucun intérêt. Elle signifie simplement que la cohérence théorique d'un modèle d'équilibre partiel ou d'équilibre général permet de faciliter l'interprétation économique des résultats obtenus en utilisant la "grille de lecture" définie par la théorie utilisée en amont. L'avantage de l'équilibre général par rapport à l'approche partielle est lié à l'exhaustivité du premier, i.e., au fait que tous les flux de l'économie considérée sont comptabilisés et toutes les réactions de ces flux modélisées. Cette exhaustivité a un coût dans la mesure où la prise en compte de toutes les interdépendances d'une économie implique qu'il est très difficile de prévoir, ex-ante, les résultats d'un scénario donné, surtout si ce dernier est complexe et correspond à des réformes touchant directement et simultanément différents secteurs d'activité, différents biens, différentes catégories de ménages, etc. ${ }^{12}$ Le recours à la théorie économique sousjacente doit alors permettre d'expliquer, ex-post, les résultats obtenus et la cohérence globale de la modélisation en équilibre général calculable permet de s'assurer de la robustesse de l'analyse. Un MEGC est, par construction, le contraire d'une boite noire. S'il est souvent perçu comme tel, c'est parce que les modélisateurs en équilibre général ne font pas l'effort suffisant pour expliquer leurs résultats en se contentant (trop souvent) d'une description linéaire des effets sans détailler les mécanismes économiques à l'œuvre.

\section{Eléments de conclusion}

En résumé, les MEGCs centrés sur l'agriculture diffèrent des modèles d'équilibre partiel multi-marchés essentiellement par leur caractère exhaustif. L'agriculture des pays développés n'occupe plus aujourd'hui qu'une part très faible de l'emploi total et elle n'a donc qu'une influence très limitée sur le coût d'opportunité du travail dans l'économie. En d'autres termes, les différents secteurs agricoles font face à une offre de travail de long terme élastique et ils sont pratiquement sans impact sur le rapport du coût du travail à celui du capital dans l'ensemble de l'économie. Les modèles d'équilibre partiel

\footnotetext{
${ }^{12}$ La même critique peut être formulée à l'encontre des "grands" modèles d'équilibre partiels multi-marchés.
} 
supposent (le plus souvent) que les offres des différents facteurs primaires adressées à l'agriculture sont ou infiniment élastiques ou totalement exogènes. La réalité se situe entre ces extrêmes, et elle peut être simulée sur la base d'analyses en équilibre général calculable sous différentes hypothèses relatives, en particulier, au fonctionnement des marchés des facteurs primaires de production ou aux règles de bouclage macro-économiques (Kilkenny, 1991).

L'intérêt essentiel des MEGCs centrés sur l'agriculture est qu'ils permettent d'étudier toutes les conséquences de scénarios de réforme des politiques agricoles dans un cadre global et cohérent sur un plan théorique. Même si l'importance de la branche agricole dans les pays développés décrô̂t régulièrement en termes de personnes occupées et de contribution au Produit Intérieur Brut, les effets de "spillover" sur les autres secteurs productifs, le poids des échanges de produits agricoles et agroalimentaires dans la balance commerciale et le coût budgétaire des dépenses en faveur de l'activité agricole justifient, dans de nombreux cas, l'étude de problèmes agricoles dans un cadre d'équilibre général calculable. De plus, un autre avantage de la modélisation en équilibre général calculable par rapport à une approche partielle centrée est que la première permet d'étudier des scénarios de réforme dans le reste de l'économie. Cette caractéristique est particulièrement importante quand l'objet d'analyse correspond à des simulations de réformes globales qui affectent l'ensemble de l'économie, du type de l'accord final de l'Uruguay Round.

Il est souvent affirmé qu'une des principales faiblesses des MEGCs centrés sur l'agriculture est le caractère trop frustre de la désagrégation en secteurs et en biens de la branche agricole, et des branches en amont et en aval (Hertel, 1990). ${ }^{13}$ La critique peut être étendue en notant que les représentations des technologies de production, des préférences des consommateurs et des instruments de politique économique (agricole notamment) sont, très souvent, trop rudimentaires pour constituer une approximation satisfaisante de la réalité. En pratique, cette critique s'applique à tout modèle appliqué insuffisamment détaillé, qu'il soit d'équilibre général ou d'équilibre partiel. Un MEGC centré sur

\footnotetext{
${ }^{13}$ Quelques exemples illustrent clairement ce point. Le modèle mondial de Trela et al. (1987) ne distingue que deux produits, le blé (tendre) et un agrégat unique regroupant tous les autres biens de l'économie américaine. Le modèle mondial de Fehr et Wiegard (1996) distingue onze pays/régions, mais considère que tous les produits agricoles peuvent être agrégés en un seul bien et que tous les produits agro-alimentaires peuvent également être agrégés en seul bien. Le modèle ECAM (European Community Agricultural Model) de Folmer et al. (1995) distingue 24 produits agricoles, mais les secteurs d'activité agroalimentaires ne sont pas modélisés de manière explicite dans la mesure où une "simple" matrice de transition est utilisée pour déterminer les quantités des différents biens agro-alimentaires produits à partir des biens agricoles différenciés dans le modèle.
} 
l'agriculture est, essentiellement, un assemblage cohérent et complet de différents modules d'équilibre partiel. Le réalisme de tout modèle relève de la responsabilité du modélisateur, et ce n'est pas un problème spécifique de l'équilibre général calculable. Néanmoins, cette critique s'applique sans doute avec plus de force dans le cas de l'équilibre général calculable en raison de l'exhaustivité de ce dernier. J. Whalley (1986) souligne, à juste titre, "the need to move from general to special-purpose models if CGE analysis is to become more policy relevant". Il ajoute que "future efforts be directed at developing special purpose models, tailored to address specific issues.

\section{Références bibliographiques}

Arrow K. J., Debreu G., 1954, Existence of an equilibrium for a competitive economy. Econometrica, 22(3), p. 265-290.

Arrow K. J., Hahn F. H., 1971, General Competitive Analysis. San Franscico, CA: Holden-Day.

Beaumais O., Schubert K., 1994, Equilibre général appliqué et environnement: de nouveaux comportements pour le consommateur et le producteur. Revue Economique, 45(3), p. 905-916.

Borges A. M., 1986, Les modèles appliqués d'équilibre général : une évaluation de leur utilité pour l'analyse des politiques économiques. Revue Economique de l'OCDE, 7, p. 7-47.

Fehr H., Wiegard W., 1996, A CGE examination of worlwide agricultural liberalisation policies: Model structure and preliminary results. In Fossati A., ed., Economic Modelling Under the Applied General Equilibrium Approach, Avebury.

Folmer C., Keyzer M. A., Merbis M. D., Stolwijk H. J. J., Veenendaal P. J. J., 1995, The Common Agricultural Policy beyond the MacSharry Reform, North Holland.

François J. F., Shiells C. R., eds., 1994, Modeling Trade Policy: Applied General Equilibrium Assessments of North American Free Trade. Cambridge University Press.

Gohin A., Guyomard H., Le Mouël C., 1998, Conséquences de modifications des niveaux et/ou des modalités d'octroi du soutien interne dans le secteur des grandes cultures, céréales et oléoprotéagineux : analyse en équilibre général. INRA-ESR, Rennes, Rapport intermédiaire pour le Ministère de l'Agriculture, de la Pêche et de l'Alimentation, DAFE. 
Hertel T. W., Thompson R. L., Tsigas M. E., 1989, Economywide effects of unilateral trade and policy liberalization in US agriculture. In Stoeckel A. B., Vincent D., Cuthbertson S., eds., Macroeconomic Consequences of Farm Support Policies, Duke University Press, Durham, N.C.

Hertel T. W., 1990, General equilibrium analysis of US agriculture: What does it contribute? The Journal of Agricultural Economics Research, 42(3), p. 3-9.

Hertel T. W., 1990, La libéralisation des échanges agricoles et les pays en développement : revue des modèles existants. Dans Goldin I. et Knudsen O., eds., Libéralisation des échanges agricoles : implications pour les pays en développement, OCDE, Paris.

Horridge M., Pearce D., 1988, Modeling the effects on Australia of interventions in world agricultural trade. IMPACT preliminary working paper, $\mathrm{n}^{\circ} \mathrm{OP}-65$, University of Melbourne.

Johansen L., 1960, A Multisectoral Study of Economic Growth. Amsterdam, North Holland

Jorgenson D. W., 1984, Econometric methods for applied general equilibrium modeling. In Scarf H.

E. et Shoven J. B., eds., Applied General Equilibrium Analysis, Cambridge, Cambridge University Press.

Kilkenny M., Robinson S., 1990, Computable general equilibrium analysis of agricultural liberalization : factor mobility and macro closure. Journal of Policy Modeling, 12, p.527-556.

Kilkenny M., 1991, Computable general equilibrium modeling of the agricultural policies: Documentation of 30 sector FPGE GAMS model of the United States. Agriculture and Rural Economy Division, USDA-ERS, Staff report n AGES9125.

Midmore P., 1987, The impact of farm prosperity on the rest of the Welsh economy. Department of Agricultural Economics and Marketing. University College of Wales.

Roberts D., :994, A modified Leontief model for analysis of the impact of milk quotas on the wider economy. Journal of Agricultural Economics, 45(1), p. 90-101.

Roningen V., 1986, A Static World Policy Simulation (SWOPSIM) modelling framework. US Department of Agriculture, ERS, Staff report n AGES860625.

Scarf H. E., 1967, The Approximation of Fixed Points of a Continuous Mapping. SIAM Journal of Applied Mathematics, 15, p. 1328-1343.

Schubert K., 1993, Les modèles d'équilibre général calculable : une revue de la littérature. Revue d'économie politique, 103(6), p. 775-825. 
Trela I., Whalley J., Wigle R., 1987, International trade in grain: Domestic policies and trade conflicts. Scandinavian Journal of Economics, 89(3), p. 271-283.

Tyers R., Anderson K., 1986, Distorsions in world food markets: A quantitative assessment. Document de référence pour le Rapport sur le développement dans le monde, La Banque Mondiale, Washington D.C.

Whalley J., 1986, Hidden challenges in recent applied general equilibrium exercices. In Piggott E. et Whalley J., eds., New Developments in Applied General Equilibrium Analysis, New York: Cambridge University Press. 
Tableau 1. L'importance des hypothèses relatives aux mobilités factorielles et aux règles de bouclage macro-économiques : illustration dans le cas d'une suppression unilatérale du programme de soutien agricole américain (source : Kilkenny et Robinson, 1990)

\begin{tabular}{|c|c|c|c|c|c|c|c|c|c|}
\hline & \multicolumn{9}{|c|}{$\begin{array}{l}\text { impacts sur les prix de marché des produits agricoles } \\
\text { (en pourcentages par rapport à la base) }\end{array}$} \\
\hline & \multicolumn{3}{|c|}{ lait } & \multicolumn{3}{|c|}{ céréales } & \multicolumn{3}{|c|}{ autres produits agricoles } \\
\hline & terre & terre-travail & total & terre & terre-travail & total & terre & terre-travail & total \\
\hline ménages & $-13,7$ & 2,4 & 8,1 & $-15,7$ & $-11,4$ & $-3,1$ & $-14,5$ & $-8,2$ & $-4,5$ \\
\hline investissement & $-14,5$ & 2,4 & 8,1 & $-16,1$ & $-11,5$ & $-3,1$ & $-15,0$ & $-8,2$ & $-4,5$ \\
\hline déficit & $-12,4$ & 3,1 & 8,1 & $-14,5$ & $-10,6$ & $-2,6$ & $-12,8$ & $-7,5$ & $-4,1$ \\
\hline moyenne & $-13,5$ & 2,6 & 8,2 & $-15,3$ & $-11,2$ & $-2,9$ & $-14,1$ & $-8,0$ & $-4,4$ \\
\hline \multicolumn{10}{|l|}{ comparaison } \\
\hline SWOPSIM & & $-6,37$ & & & $-21,32$ & & & $-12,1$ & \\
\hline
\end{tabular}


